\title{
PROPUESTA METODOLOGICA PARA EL ANALISIS DE LAS ACTIVIDADES EN LOS LIBROS DE TEXTO DE CIENCIAS NATURALES
}

\author{
PROPOSTA METODOLÓGICA PARA A ANÁLISE DE ATIVIDADES EM \\ TEXTBOOKS DE CIÊNCIA NATURAL
}

\author{
Adriana Gómez-Jiménez \\ Ministerio de Educación Pública de Costa Rica \\ Profesora de Ciencias Naturales
}

Silvia Porro
Universidad Nacional de Quilmes, Argentina
Departamento de Ciencia y Tecnología

Manuel Sandoval-Barrantes

Universidad Nacional de Costa Rica

Escuela de Química

\section{Resumen}

Este artículo realiza una propuesta metodológica para analizar las actividades que se encuentran en los libros de texto de ciencias naturales de secundaria en el contexto de Costa Rica. Mediante una revisión teórica se realiza una metodología que permite categorizar, clasificar y analizar las actividades, utilizando técnicas cualitativas y cuantitativas que permitan generar resultados acerca de las actividades que promueven los libros de texto. Para la recolección de la información cualitativa se propone realizar una investigación de documentos importantes de la política educativa, y para el análisis cuantitativo se propone utilizar tablas que funcionan como fichas de recolección de información para cada dimensión del análisis gestionando una matriz de datos que sirva para un análisis estadístico que conlleva al análisis mediante una triangulación de datos.

Palabras clave: Actividades. Libros de Texto. Ciencias Naturales. 


\section{Resumo}

Este artigo faz uma proposta metodológica para analisar as atividades encontradas nos livros didáticos de ciências naturais do ensino médio no contexto da Costa Rica. Através de uma revisão teórica, é realizada uma metodologia que permite categorizar, classificar e analisar as atividades, utilizando técnicas qualitativas e quantitativas que permitem gerar resultados sobre as atividades promovidas pelos livros didáticos. Para a coleta de informações qualitativas, propõe-se realizar uma investigação de documentos importantes da política educacional e, para a análise quantitativa, propõe-se a utilização de tabelas que funcionem como cartões de coleta de informações para cada dimensão da análise, gerenciando uma matriz de dados que sirva para uma análise estatística que leva à análise através de uma triangulação de dados.

Palavras-chave: Atividades. Livros Didáticos. Ciências Naturais.

\section{Introducción}

Los libros de texto (LT) se caracterizan por incluir contenido didáctico y actividades. Las actividades promueven en el estudiantado aprendizajes más significativos permitiendo comprender el contenido didáctico. Las actividades se implementan en las clases de ciencias mediante la guía del docente y muchas veces acompañados del apoyo didáctico de los LT. Al haber muchas actividades con diversas finalidades, en este estudio solo se abordarán las actividades propuestas en los LT de ciencias naturales en el contexto de Costa Rica.

Algunos autores se refieren a las actividades llamándolas estrategias metodológicas (MASSA, 2015); tareas (MALHUE; MORAGA; LAZO, 2011; LEYMONIÉ, 2009), actividades de enseñanza y aprendizaje (VILLALOBOS, 2003) o solo actividades de aprendizaje (PENZO et al., 2010), debido a que el término es ambiguo, esta investigación considera que no hay diferencia entre esos términos y se referirá a las mismas solo con el término "actividades" haciendo alusión a los términos expuestos anteriormente.

La propuesta aborda un análisis en las actividades de los LT, planteando la implementación de estudios como este desde dos perspectivas; la primera corresponde a un estudio sobre el tratamiento de los LT en su contexto, mediante un análisis cualitativo; la segunda, es la realización de un análisis cualitativo y cuantitativo de las actividades, que 
desde lo didáctico conllevan a procesos de recolección, tratamiento y análisis de diversos datos para cada perspectiva (contextual y didáctico).

\section{Antecedentes del estudio de los libros de texto}

Actualmente hay una gran diversidad de LT a disposición de los docentes y del estudiantado, que han sido incorporados a través de los años como instrumentos o herramientas para el uso tanto dentro como fuera del contexto educativo, esto ha contribuido en el proceso de enseñanza y aprendizaje durante mucho tiempo. En este sentido, al abordarse el LT como material directo de referencia para tener acceso a la muestra de actividades, se considera necesario tener un panorama aproximado sobre el tipo de investigaciones que se han realizado en torno al tema.

Por primera vez en la lengua inglesa en 1750 aparece el término "libro escolar" (Schoolbook), posteriormente en 1830 se refieren a este objeto como LT (Textbook). En 1992, el francés Alain Choppin, investigador reconocido a nivel internacional por sus investigaciones relacionadas a los LT, propone una tipología de los mismos y se refiere a estos como manuales escolares, con la finalidad de que al ser utilizados en una clase sirvan de apoyo en la enseñanza de una determinada disciplina; el mismo autor se refiere a "obras para escolares" las cuales también son materiales de apoyo y tienen la finalidad de reforzar, resumir y ejercitar ciertos contenidos.

En España y Portugal se utiliza la terminología "manuales escolares" para referirse a una palabra que en Iberoamérica nos referimos como "libros de texto". Ossenbach y Somoza (2009), investigadores de los manuales escolares en América Latina, mencionan que en estos lugares se utilizan términos como: libros, texto, manuales, acompañados de adjetivo "escolar" para referirnos al mismo objeto. Estos autores mencionan que "los libros escolares son un fenómeno pedagógico, pero también cultural, político, administrativo, técnico y económico.” (p. 15). De ahí que la edición de los LT, tanto de su contenido como de las actividades que contiene, depende no solo del sistema político sino además de todos los demás sectores que conforman la sociedad, estos son contextualizados según las normas establecidas en cada sector.

Autores de gran peso académico relacionados con los LT (CHOPPIN, 2001; OSSENBACH; SOMOZA, 2009; CARBONE, 2003) argumentan la importancia de más investigaciones relacionadas a los LT y concuerdan con que estos son una herramienta que sigue siendo utilizada en las instituciones educativas porque es un instrumento que permite la socialización, la comunicación, además de que promueve la cultural.

Otros estudios relacionados a la investigación en LT de ciencias naturales (FARÍAS; CASTELLÓ; MOLINA, 2013; LÓPEZ; POSTIGO, 2014) mencionan que este objeto es un recurso 
didáctico muy utilizado por el profesorado, además de ser el más aceptado por el resto de la comunidad educativa.

En Costa Rica, la historia de la educación registra desde 1730 la implementación de LT en la enseñanza mediante las cartillas (silabario) y el catón (lecturas con base en el cristianismo); estos eran importados y distribuidos a distintas escuelas (MOLINA, 2016). A partir de ese hecho, se registran otros momentos en los que el LT está presente en la educación costarricense, sin embargo, se da énfasis a los últimos tres momentos históricos (ver Tabla 1), debido a que son los más significativos para la investigación, por ser los más recientes.

Los LT en este contexto fueron materiales de distribución gratuita para el estudiantado, al menos en los dos primeros programas.

Tabla 1. Programas relacionados con los LT en la historia de la educación de Costa Rica

\begin{tabular}{clll}
\hline Año inicio & Programas & $\begin{array}{l}\text { Cobertura } \\
\text { Primaria }\end{array}$ & $\begin{array}{c}\text { Cobertura } \\
\text { Secundaria }\end{array}$ \\
\hline $\mathbf{1 9 6 0}$ & Odeca-Rocap & Abundante & Escasa \\
\hline $\mathbf{1 9 8 4}$ & Hacia la Luz & Abundante & Sin registro \\
\hline $\mathbf{1 9 9 4}$ & Hacia el siglo XXI & Abundante & Regular \\
\hline
\end{tabular}

Fuente: Elaboración propia a partir de las referencias bibliográficas.

El programa Odeca-Rocap consistió en "un plan para la unificación educativa de Centroamérica, hecho con la colaboración del Gobierno de los Estados Unidos a través de la ROCAP (Regional Office for Central América and Panamá), la cooperación de la ODECA, los ministerios de educación de los países del istmo y las misiones de la AID (Agency for International Development), del Departamento de Estado de los Estados Unidos en los países de Centroamérica.” (OVARES, 1977, p. 13). Aproximadamente entre 1960 y 1970 fueron implementados en la educación costarricense, pero al ser libros poco contextualizados eran muy criticados. La importancia en este punto consiste en la distribución de LT a todas las escuelas del país, para la educación secundaria eran escasos los LT.

El según momento en el que los LT están presentes en la historia de Costa Rica es en el surgimiento del "Programa Hacia la luz" que a partir de 1984 sustituye al programa anterior, los LT se distribuyen a todas las escuelas públicas de Costa Rica, pero a pesar de ese hecho, dejaron de reeditarse.

En un tercer momento, la presencia de los LT se da al crearse el "Programa Hacia el Siglo XXI" de la política educativa implementada en 1995 y que se inició con ediciones de LT para todos los niveles educativos, hechos por profesionales costarricenses. Para Gallardo (2001, p. 83), “esta serie 
está integrada por un conjunto denominado "Complejo Didáctico", que incluye el libro de texto, un cuaderno de actividades (solo para primaria) y una guía para el docente". La distribución de LT a todas las escuelas primarias se implementó por varios años hasta que se descontinuaron, esta política educativa estuvo con vigencia hasta el curso lectivo 2016. Actualmente en Costa Rica se encuentran LT de distribución privada, que son los que lideran el mercado de textos escolares del país, los cuales son utilizados como recurso de apoyo tanto en el sector público como en el privado.

La historia da evidencia sobre las intenciones que promueven los LT de acuerdo a las políticas educativas, este recurso educativo varía de acuerdo a esas políticas, por eso la importancia de involucrar los antecedentes de los LT ya que estos dependen de la organización política educativa del país. A continuación, se presenta las políticas educativas según el contexto.

\section{Las políticas educativas y curriculares del contexto}

A manera deductiva se inicia por las políticas educativas y curriculares, que son una acción del estado relacionadas al sistema educativo y que conllevan a un cambio en el actuar educativo con el fin de mejorar la enseñanza y el aprendizaje, y a la vez son un reflejo de la necesidad que tienen las nuevas generaciones de encontrar un aprendizaje con más significado. En muchos contextos educativos el docente tiene la función de ser un guía en el aprendizaje de los contenidos, para el contexto costarricense se ha implementado el socioconstructivismo como apoyo para este enfoque, por este motivo se menciona en el programa de estudios los aportes de referentes teóricos de gran peso como Jean Piaget, David Ausubel, Jerome Bruner, Lev Vygotsky, Henri Wallon y Paulo Freire (MINISTERIO DE EDUCACIÓN PÚBLICA, 2017).

Recientemente una investigación doctoral relacionada al estudio de las actividades en LT (SÁEZ, 2016) menciona que en la región hay un interés en el avance de las políticas educativas, tecnológicas y de innovación en el que se aspira a generar conocimiento científico y tecnológico propio, que ayude a solucionar los problemas sociales. Dicho interés refuerza la necesidad de una revisión de la documentación oficial educativa que conlleve a encontrar el planteo implícito de lo que deberían proponer las actividades en los LT, estas a la vez reflejan una intencionalidad política, social y cultural, que son transmitidas al estudiantado. Tomando en cuenta que los LT dirigidos al sector educativo reflejan la política curricular educativa de un país, se parte del hecho que el contenido didáctico es el mismo, lo que cambia en cada contexto es la forma en la que se presentan las actividades y, por ende, como el docente las aborda con el apoyo del LT.

A manera de ejemplo, entre las políticas educativas y curriculares del contexto en Costa Rica se encuentra la ley fundamental de educación, que involucra derechos, deberes, alcances y organización de la educación, la política educativa vigente hasta el 2016, llamada hacia el siglo XXI,

Revista RBBA $\mid$ Revista Binacional Brasil Argentina 
definida en su momento como la ley marco del sistema educativo costarricense, después de la Constitución Política, y tiene como objetivo la adecuación de la disposición constitucional a la realidad específica del país. Una manera de analizar si dicha política educativa se ha cumplido o se está cumpliendo, es analizar las actividades propuestas en los LT de ciencias naturales y relacionarlas con los planteamientos de dicha política educativa que estuvo vigente hasta el 2016.

De la política educativa se plasma el programa de estudios, que constituye "la columna vertebral del quehacer educativo, y deben responder a la formación de una ciudadanía consciente de sus deberes y responsabilidades con el progreso nacional" (MINISTERIO DE EDUCACIÓN PÚBLICA, 2005, p. 1), a partir de este documento, los docentes realizan su planteamiento didáctico del cual se derivan las actividades que muchas veces son tomadas de los LT.

Las políticas educativas y curriculares del contexto, tienen una intención en la implementación de una educación científica congruente con las tendencias actuales de acuerdo con cada contexto, direccionada a una educación en que la ciencia sea más aplicada a la vida y que el estudiantado sea capaz de utilizar los aprendizajes obtenidos para mejorar su calidad de vida, la de su entorno y la del mundo entero. En este sentido, las actividades que se proponen en los LT ajustadas a la realidad inmediata del estudiantado son clave para desarrollar diversas habilidades, capacidades y destrezas científicas.

La descripción deductiva llevada a cabo anteriormente, da pie para avanzar al objeto central de estudio, las actividades.

\section{Las actividades como objeto de estudio: modelos científicos escolares}

Las actividades incluidas en los LT, pueden estar en cualquier parte de la unidad didáctica, es decir, se encuentran al iniciar o terminar un tema o contenido didáctico. Por lo tanto, el análisis de las actividades depende mucho del tema que se seleccione. Muchas veces las actividades en los LT incluyen representaciones gráficas que no necesariamente son las más idóneas para ejemplificar cada uno de los modelos científicos escolares (ADÚRIZ-BRAVO et al., 2014). Si bien el tema es bastante amplio y por lo tanto no se abarcará en este estudio, cabe aclarar que las actividades que proponen los LT muchas veces presentan modelos científicos escolares para que el estudiantado "modelice" determinado hecho o concepto, de ahí la importancia de la exposición del tema en este punto, ya que también se pueden analizar las actividades desde otra mirada.

Penzo et al. (2010, p. 9) mencionan que las actividades de aprendizaje "sirven para aprender, adquirir o construir el conocimiento disciplinario propio de una materia o asignatura; y para aprenderlo de una determinada manera, de forma que sea funcional, que pueda utilizarse como instrumento de razonamiento." la ayuda que obtienen el estudiantado con las actividades genera la 
construcción de un conocimiento de la ciencia a partir de las representaciones que se le presentan, pero a menudo solo se les muestra un modelo de ciencia ya terminado más no su proceso (RAMÍREZ; FLEISNER; VIERA, 2017), se les presenta como un producto verdadero, por lo que esto tiene consecuencias erróneas en el aprendizaje, y en la enseñanza de ese contenido, esto repercute en el diseño de las actividades, ya que éstas serán ricas solamente en la parte teórica, sin ningún tipo de significado en el contexto del estudiante (QUINTANILLA; CUELLAR; CAMACHO, 2008), ya que solo se enfocarían en lo memorístico.

Con lo expuesto anteriormente, se puede construir una definición del LT, aunque como explica Choppin (2001) esto resulta en una tarea difícil. Por comodidad se definirá el LT a partir de los autores citados anteriormente, y para fines escolares se entenderá que el LT es una construcción tanto física como de contenidos didácticos, de los saberes que se quieren comunicar a una determinada población estudiantil, los cuales son reafirmados mediante las actividades que se realizan y que tienen como finalidad un aprendizaje más significativo.

Algunos autores piensan que el uso de modelos en la explicación de una teoría científica es uno de los tantos problemas epistemológicos que tiene la didáctica de las ciencias, ya que los modelos deben comprenderse como mediadores entre la ciencia y la realidad (ADÚRIZ-BRAVO; LABARCA; LOMBARDI, 2014). También Concari (2001, p. 1) explica que los modelos son entendidos como una representación de una "situación real de manera incompleta, aproximada e inexacta...". Lo que menciona la autora puede generar alguna discusión desde lo epistemológico, pero concuerda con lo expuesto por Adúriz-Bravo et al. (2014).

Adúriz-Bravo et al. (2014, p. 42) mencionan además que "un modelo no es verdadero, sino similar al sistema real en algunos aspectos y grados, que dependen tanto de las capacidades biológicas del ser humano como de convenciones y paradigmas socialmente aceptados.”. Los mismos autores se refieren al modelo como mediador, cuya relación es la siguiente: teoría $\leftrightarrow$ modelo $\leftrightarrow$ realidad, y mencionan que el modelo es "un objeto abstracto, conceptualmente construido, en el cual se consideran como variables solo los factores relevantes..." además enfatizan en que “...la función principal de los modelos como mediadores entre teoría y realidad consiste en permitir el uso de una teoría científica para la explicación de un fenómeno natural a través de una conceptualización de tal fenómeno." (ADÚRIZ-BRAVO et al., 2014, p. 42)

La idea de modelo mediador, según los autores mencionados, facilita la enseñanza porque permite dejar atrás la repetición y ayuda al docente en el proceso de manipulación del mismo, es decir, permite poder pensar sobre hechos claves que dieron origen a cada modelo atómico. En este sentido, la realidad de ese modelo mediador también esta mediada por las actividades que proponen los docentes en sus diferentes recursos didácticos. 
Con el mismo sentido lo expresan Lucas y Martínez (2016, p. 1) al formular que "los filósofos de la ciencia han comenzado a reconocer en los modelos un recurso metodológico fundamental e indispensable de la ciencia moderna", un ejemplo de eso es el estudio de Adúriz-Bravo et al. (2014), gracias a este tipo de investigaciones, los modelos se articulan en la mediación didáctica y, en consecuencia, en la elección de LT por parte del docente.

Tanto en el contenido didáctico como en las actividades se pueden encontrar los modelos representados con imágenes, que forman parte del discurso en el proceso de enseñanza y aprendizaje de las ciencias. En este sentido, la teoría atómica utiliza los modelos para fundamentar la teoría científica que el estudiantado requiere aprender para el dominio del tema.

Los LT son materiales construidos con el propósito de ayudar en la tarea docente y apoyar al estudiantado en la construcción de nuevos conocimientos, si bien hay docentes de ciencias naturales que guían el conocimiento del alumnado sin necesidad de un LT, también hay docentes que optan por utilizar el LT como recurso didáctico, apoyándose en las actividades contenidas en los mismos, estos al contener representaciones gráficas más el contenido didáctico de un determinado tema, favorecen al estudiantado a comprender con mayor practicidad el modelo científico escolar que se pretende tanto el contenido didáctico como en la realización de las actividades propuestas en los LT.

\section{Teorías relacionadas con el aprendizaje en el enfoque curricular}

Existe una preocupación por mejorar la práctica docente (MASSA, 2015), ya que el profesorado es el único que puede mejorar la práctica educativa; una de tantas implicaciones es la reflexión de las actividades que promueven los LT, y se puede realizar mediante la caracterización de las mismas a partir de los diferentes aportes teóricos expuestos por investigadores e investigadoras de gran trayectoria. La finalidad es obtener un panorama más amplio de las actividades para así lograr orientar la investigación metodológica.

En este sentido, los modelos de enseñanza, a pesar de ser un campo de gran amplitud teórica, no se pretende en este trabajo abordar la totalidad de los mismos, sino solamente introducir algunos aspectos en relación con las actividades de enseñanza y aprendizaje, lo cual se muestra en la Tabla 2. 
Tabla 2. Aportes teóricos relacionados con las actividades

\begin{tabular}{ll}
\hline Investigadores & Aportes teóricos \\
\hline Jean Piaget & Las actividades deben \\
& - enriquecer la crítica, la voluntad y la motivación del estudiantado. \\
& - promover la investigación propia, siendo estas más enriquecedoras. \\
& - enriquecer el significado del contenido que se enseña frente al significante \\
& (expresión verbal o simbólica). \\
\hline David Ausubel & Los materiales de apoyo (en este caso, actividades) deben \\
& - estar lógicamente organizados. \\
& - relacionarse con los contenidos previos del estudiantado. \\
& nueva información. \\
& Las actividades deben \\
& - permitir el desarrollo de competencias sobre sus propios conocimientos. \\
& - promover una investigación participativa y actividades en las que se construya \\
un conocimiento integrado. & - buscar responder a preguntas o conflictos existenciales.
\end{tabular}

Lev Vygotsky Las actividades deben

- promover situaciones de socialización.

- facilitar la problematización, que permita la reconstrucción cognitiva.

Henri Wallon Las actividades deben

- presentarse de forma atractiva, de acuerdo con el interés del estudiantado.

- plantearse con diferentes niveles de dificultad.

- aplicarse a situaciones fuera del contexto escolar.

- favorecer la socialización.

Paulo Freire Las actividades deben

- promover una participación activa, creativa y responsable.

- facilitar la resolución de problemas reales.

- plantearse para grupos pequeños con el fin de fomentar el cooperativismo y la interacción.

Fuente: Elaboración propia. 
Dichos autores son los referentes propuestos en el programa de estudios que se analiza, estos han teorizado sobre el aprendizaje, el cual es, según los mismos, un proceso de construcción que será útil en el futuro de cada estudiante. En relación con las actividades, cada uno de los autores mencionados caracteriza las actividades, pero en general éstas deben ajustarse al contexto, ser atractivas para el estudiantado y que a la vez propicien la problematización con el objetivo de que el alumnado practique el razonamiento y el análisis y que esto le ayude en su vida cotidiana.

Del planteo anterior, se desprende la metodología del presente estudio, la cual se basa en el marco teórico expuesto en esta sección.

\section{Metodología}

Este estudio presenta las características de una investigación descriptiva, ya que es nueva en su contexto, y es base de la investigación correlacional que a la vez proporciona información explicativa y es un estudio altamente estructurado (HERNÁNDEZ; FERNÁNDEZ; BATISTA, 2010).

Luego de la elección del tema, la construcción de la propuesta para analizar las actividades en los LT se realiza mediante la búsqueda de fuentes relacionadas a los procesos de elaboración de propuestas para realizar una investigación (IGLESIAS, 2015; MARRERO, 2012; HERNÁNDEZ, FERNÁNDEZ; BATISTA, 2010; GÓMEZ, 2012; SOUZA, 2009; MORA, 2005), de forma contextualizada se ajustaron al objetivo del estudio siguiendo un proceso sistemático que generó una guía metodológica para analizar las actividades en los LT. Esta se observa en la Tabla 3.

Tabla 3. Guía metodológica para analizar las actividades en LT

\begin{tabular}{lll}
\hline Estructura & \multicolumn{2}{c}{ Sistematización de la investigación } \\
\hline Introducción & - Justificación de la importancia del análisis de las actividades en los LT. \\
& - Descripción de las concepciones y caracterización del LT. \\
& LT. & Exposición de los antecedentes nacionales e internacionales sobre investigaciones en \\
& - Relación de los LT con las políticas educativas y curriculares de la educación. \\
& - Contextualización del LT en la educación. \\
& - Descripción contextualizada de las actividades utilizadas en los LT \\
& LT. & \\
& - Delación de los aprendizajes cognitivos con lo que promueven las actividades en los \\
Metodología & - $\quad$ Claboración del problema y contextualización. \\
& - Descripción de los criterios de selección de las muestras. \\
& - Presentación de las técnicas de recolección de datos. \\
\hline
\end{tabular}




\begin{tabular}{ll}
\hline & Descripción de los instrumentos de recogida de información. \\
& - Descripción de los procesos de validación de la investigación. \\
& - Explicación del análisis y tratamiento de los datos. \\
\hline Resultados & Presentación de los hallazgos de la investigación de acuerdo a las muestras \\
& seleccionadas y relacionados con los objetivos de la investigación. \\
& Presentación estructural de los datos, acompañados de tablas, gráficos que faciliten la \\
& exposición y la lectura. \\
\hline Discusión & Interpretación de los resultados relacionándolos con los objetivos de la investigación. \\
& - Interpretación de los resultados relacionándolos con el marco teórico de la \\
\hline Conclusión & investigación. \\
\hline
\end{tabular}

A partir de lo expuesto en la Tabla 3 y para cumplir con el objetivo de esta investigación, se presentan en la siguiente sección los resultados seguido de una discusión. Los resultados se exponen de acuerdo a lo estipulado en esta sección, de manera descriptiva, enfatizando en los procesos que se llevaron a cabo de acuerdo con la propuesta metodológica. Lo que se presenta a continuación surge a partir de la información del marco teórico y metodológico, además de la revisión y contextualización del objeto de estudio. Para facilitar el análisis la información se organizó en dos niveles: el nivel contextual que corresponde al proceso de investigación documental, y el nivel didáctico que se relaciona con el análisis de las actividades en los LT.

Las técnicas de recolección de datos en este punto se basan en lo propuesto por la investigación documental (GÓMEZ, 2012), que a partir del análisis de los documentos citados permiten obtener datos relacionados a los LT en el contexto; los instrumentos utilizados para realizar este proceso consisten en fichas de identificación de documentos y de investigación mixta, las cuales se realizaron en el programa de análisis cualitativo ATLAS.ti, el cual es utilizado como gestor de fichas. Para cumplir con el proceso de calidad de las fuentes, la autora Pinto (2015), propuso criterios e indicadores sobre la calidad y evaluación de las fuentes utilizadas, en la cual se revisa la autoría, la actualización, el tipo de contenido, la accesibilidad, la funcionalidad, la navegabilidad y el diseño de las fuentes de información.

La matriz de datos obtenida a partir del proceso de análisis se realizó en el programa estadístico SPSS; se presentarán los datos numéricos que darán evidencia, a partir del análisis por medio de la triangulación de datos, de lo que promueven las actividades en los LT, y si éstas están relacionadas con el programa de estudio y los demás documentos analizados. Esta técnica de análisis permite evaluar qué tipo de aprendizajes adquiere el estudiantado mediante las actividades propuestas por los LT. 


\section{Resultados}

Propuesta metodológica para el análisis de las actividades presentes en los libros de texto

Tipo: metodología estructurada en dos dimensiones: contextual y didáctica, cada una con sus pasos respectivos.

Objetivo: Analizar las actividades de enseñanza y aprendizaje que promueven los libros de texto de ciencias naturales en un determinado tema.

Alcance: Diseñada para ser aplicada por docentes de ciencias naturales para libros de texto de secundaria en un tema determinado.

Primer paso: Selección de la muestra y criterios de selección

\section{Nivel contextual}

En esta sección se describe el corpus documental a utilizar para realizar la investigación documental, necesario para contextualizar posteriormente el análisis. Los documentos recomendados a utilizar son:

- Ley Fundamental de Educación

- Política Educativa

- Lineamientos específicos de evaluación del Ministerio de Educación Pública del país

- Programa de estudio de ciencias naturales de acuerdo al tema en estudio

Estos documentos forman parte del currículo educativo del contexto en el que se encuentran los LT, para seleccionar los documentos se tomó en cuenta aspectos como el contexto, el contenido, las fuentes y la vigencia de toda la muestra documental.

\section{Nivel didáctico}

La muestra de LT corresponderá a LT de secundaria de acuerdo a las políticas educativas del país en cuanto al uso de los mismos en los espacios educativos, además se debe contemplar que los mismos deben contener el tema en estudio. 
Esta propuesta puede contener LT del contexto educativo relacionados a las ciencias naturales en secundaria, se seleccionarán tomando en cuenta el contexto, el nivel y ciclo educativo, los objetivos del programa de estudios de ciencias naturales de acuerdo al contenido o tema seleccionado, la propuesta de actividades de los LT y la vigencia de los mismos. El muestreo de LT será seleccionado de acuerdo a los resultados de la aplicación de un cuestionario a la población docente. Como ejemplo se puede mencionar el estudio realizado a una muestra de docentes de ciencias naturales del contexto educativo costarricense (GÓMEZ, PORRO; SANDOVAL, 2018).

Segundo paso: Selección de las técnicas de recolección de datos, los instrumentos y su validación

\section{Nivel contextual}

Este tipo de investigación implicará una recolección de datos desde el primer momento de búsqueda de información, estará sujeta a la técnica de investigación documental, la cual se llevará a cabo mediante una lectura sistemática que permitirá identificar y analizar, reflexionar e interpretar las fuentes documentales relacionadas al sistema educativo costarricense.

\section{Nivel didáctico}

La recogida de datos en este apartado se obtendrá a través de la observación de las actividades propuestas en los LT y de la investigación documental de estudios relacionados (Sáez, 2016; Malhue, Moraga y Lazo, 2011, a partir de lo anterior, se construirán las dimensiones de análisis que se utilizarán para analizar las actividades propuestas en los LT.

Tercer paso: Tratamiento de los datos y su análisis

\section{Nivel contextual}

Los datos que se utilizarán corresponderán a variables cualitativas, que pueden ser gestionadas en una matriz de datos, que permitirá describir y explicar el contenido de los documentos. El análisis de los documentos oficiales se realizará mediante un enfoque interpretativo basado en un determinado marco teórico.

\section{Nivel didáctico}

Los datos utilizados corresponderán a variables cualitativas de nivel de medición nominal. Dichos datos serán vaciados en una matriz. Este trato de las variables permitirá hacer clasificaciones (Camarero y otros, 2013) de las actividades propuestas en los LT. 
A continuación, se presenta un resumen de la propuesta metodológica detallada anteriormente, que se realizó con ayuda de la guía metodológica expuesta en la sección anterior, resultando un proceso sistemático que puede ser replicado para estudios posteriores, y para otras áreas de estudio; esto se visualiza en la Tabla 4.

Tabla 4. Niveles y estrategias metodológicas para analizar las actividades en LT

\begin{tabular}{|c|c|c|}
\hline Estrategias/Niveles & Contextual & Didáctico \\
\hline Selección de la muestra & $\begin{array}{l}\text { Documentos del currículo } \\
\text { educativo del país. }\end{array}$ & Libros de texto seleccionados \\
\hline Criterios de selección & $\begin{array}{l}\text { Se toma en cuenta el contexto, el } \\
\text { contenido, las fuentes y la vigencia } \\
\text { de los documentos. }\end{array}$ & $\begin{array}{l}\text { Se toma en cuenta el contexto, el } \\
\text { ciclo educativo, los objetivos del } \\
\text { programa de estudio, la propuesta } \\
\text { de actividades y la vigencia. }\end{array}$ \\
\hline Técnicas de recolección de datos & Investigación documental & $\begin{array}{l}\text { Observación cuantitativa de las } \\
\text { actividades. }\end{array}$ \\
\hline Instrumentos utilizados & $\begin{array}{l}\text { Fichas de identificación } \\
\text { Fichas de investigación mixta }\end{array}$ & $\begin{array}{l}\text { Cuestionario } \\
\text { Guía de valoración de las } \\
\text { actividades }\end{array}$ \\
\hline Procesos de calidad y validación & $\begin{array}{l}\text { En este nivel se toma en cuenta la } \\
\text { calidad de las fuentes utilizadas. }\end{array}$ & $\begin{array}{l}\text { En este nivel se validaron los } \\
\text { instrumentos por juicio de } \\
\text { expertos. }\end{array}$ \\
\hline Tratamiento de los datos & $\begin{array}{l}\text { Se utiliza el programa de análisis } \\
\text { cualitativo ATLAS.ti como gestor } \\
\text { de las fichas para el análisis. }\end{array}$ & $\begin{array}{l}\text { Se utiliza el programa estadístico } \\
\text { SPSS para analizar las actividades. }\end{array}$ \\
\hline Análisis de los datos & \multicolumn{2}{|c|}{$\begin{array}{l}\text { Se triangulan los datos de: nivel contextual, nivel didáctico y marco } \\
\text { teórico. }\end{array}$} \\
\hline
\end{tabular}

Fuente: Elaboración propia

\section{Conclusiones}

El hecho de analizar las actividades en los LT implica que la metodología a utilizar para tal análisis pueda ser replicada, estudiada y utilizada en otras actividades propuestas en LT de ciencias naturales.

Se puede decir que el LT, a pesar de sus diversas características apoyadas por los autores expuestos, se ha percibido a lo largo del tiempo como una "receta didáctica" (MASSA, 2015), sin embargo, la simplicidad y practicidad a la hora de su manipulación en las aulas son unas de las propiedades más importantes que lo hacen estar siempre presente. La utilización del LT en las clases 
no implica que éste sea estrictamente seguido por el docente, ya que es una herramienta didáctica que puede ser adecuada a cada entorno.

Los LT al igual que la política educativa contribuyen a la socialización, en este caso, de las ciencias naturales. Es así como las actividades de los LT se vuelven un reflejo intencionado de la política educativa, en la cual el docente establece el vínculo con los componentes de la educación costarricense mediante una transposición didáctica. Las actividades que se proponen en los LT ajustadas a la realidad inmediata del estudiantado son clave para desarrollar diversas habilidades, capacidades y destrezas científicas.

Para llevar a cabo el análisis de las actividades, primeramente, se obtendría una muestra de LT, con ello el análisis se dividirá en dos fases: la primera (contextual) corresponde a la investigación de algunos documentos oficiales, lo que permite confrontar los resultados. La segunda fase (didáctica) implica un análisis de los LT en el que se identifican las actividades.

La propuesta metodológica obtenida contribuye al conocimiento en la didáctica de las ciencias experimentales en cuanto a que cualquier docente pueda replicar la técnica para poder analizar las actividades que se encuentran en los LT, y a partir de eso el profesorado tenga criterio para evaluar las actividades que serán utilizadas en las clases de ciencias naturales y específicamente aquellas que promueven los LT.

Esta propuesta se pone a disposición de todos los profesores de ciencias naturales de secundaria de cualquier contexto, constituye una herramienta útil que permite realizar una valoración más objetiva de las actividades que proponen los libros de texto de acuerdo con las políticas educativas del cada país.

De todo el proceso anterior, se lograría sistematizar el proceso para analizar las actividades en los LT, utilizando las dos fases propuestas, y para cada una, las estrategias son consecutivamente similares. El analizar las actividades en los LT implica primero analizar el contexto contrarrestando los datos cualitativos con los datos cuantitativos y una investigación documental.

\section{Referencias}

ADÚRIZ-BRAVO, A.; LABARCA, M; LOMBARDI, O. Capítulo 2. Una noción de modelo útil para la formación del Profesorado de Química. En Merino, C., Arellano, M., y Adúriz Bravo, A. (Eds.) Avances en Didáctica de la Química: Modelos y lenguajes. (pp. 37 - 50). Ediciones Universitarias de Valparaíso. Pontificia Universidad Católica de Valparaíso. 2014.

CARBONE, G. Libros escolares: una introducción a su análisis y evaluación. Buenos Aires: Fondo de Cultura Económica. 2003.

CAMARERO, L., AlMAZÁN, A., ARRIBAS, J., M., MAÑAS, B., VALlEJOS, F., A. Estadística para la Investigación Social. 1 ed. Alfaomega. México. 2013. 
CHOPPIN, A. "Pasado y Presente de los Manuales Escolares", en Revista Educación y Pedagogía. 13, 29-30. Medellín, Universidad de Antioquia. 2001.

CID M., R.; DASILVA A., G. Estudiando cómo los modelos atómicos son introducidos en los libros de texto de Secundaria. Revista Eureka sobre Enseñanza y Divulgación de las Ciencias, 9(3), 239-337. 2012.

CONCARI, S. B. Las teorías y modelos en la explicación científica: implicancias para la enseñanza de las ciencias. Ciência \& Educação, 7(1), 85-94. 2001.

FARÍAS C., D., CASTELLÓ E., J.; MOLINA C., M. Análisis del enfoque de historia y filosofía de la ciencia en libros de texto de química: el caso de la estructura atómica. Enseñanza de las ciencias: Revista de investigación y experiencias didácticas, 31(1), 115-113. 2013.

GALLARDO. I. Una aventura educativa: el uso del libro de texto hacia el siglo XXI. Revista Educación. 25(1). pp. 81-93. 2001. Recuperado el 20/01/2017 de http://www.redalyc.org/pdf/440/44002508.pdf

GÓMEZ, B., S. Metodología de la investigación. 1 ed. Tercer Milenio. México. 2012.

GÓMEZ, J., A., PORRO, S.; SANDOVAL, B., M. Los libros de texto como recursos didácticos en la enseñanza de las ciencias naturales: El caso de modelos atómicos. Comunicación oral en IV Congreso Latinoamericano de Investigación en Didáctica de las Ciencias Experimentales. San José, Costa Rica. 2018.

GÓMEZ, J., A.; PORRO, S. Investigaciones centradas en el análisis de las actividades de aprendizaje en los libros de texto. Revista de Enseñanza de la Física. 28 (Extra), 85-90. 2016.

HERNÁNDEZ, R., FERNÁNDEZ, C.; BAPTISTA, P. Metodología de la investigación. México DF: McGrawHill. 2010.

IGLESIAS, M., E. Metodología de la investigación científica: diseño y elaboración de protocolos y proyectos. 1 ed. Centro de Publicaciones Educativas y Material Didáctico. Ciudad Autónoma de Buenos Aires. 2015.

LEYMONIÉ, J. Aportes para la enseñanza de las Ciencias Naturales. Segundo Estudio Regional Comparativo y Explicativo. Organización de las Naciones Unidas para la Educación la Ciencia y la Cultura. Oficina Regional de Educación para América Latina y el Caribe. Santiago, Chile. 2009. Recuperado de http://unesdoc. unesco. org/images/0018/001802/180275s. pdf

LÓPEZ V., D.; GUERRA R., M. Análisis de las actividades de aprendizaje incluidas en libros de texto de ciencias naturales para educación primaria utilizados en México, Enseñanza de las Ciencias, 31(2), 173-191. 2013.

LÓPEZ, M., A.; POSTIGO A., Y. Análisis de las imágenes del cuerpo humano en libros de texto españoles de primaria. Enseñanza de las Ciencias, 32(3), 551-570. 2014.

LUCAS ACCORINTI, H.; MARTÍNEZ GONZÁLEZ, J. C. Acerca de la independencia de los modelos respecto de las teorías: un caso de la química cuántica. Theoria. Revista de Teoría, Historia y Fundamentos de la Ciencia, 31(2), 225-245. 2016. 
MALHUE, S., R., MORAGA, C., M.; LAZO, S., L. Análisis Taxonómico de los Libros de Texto para la Enseñanza de Química en Educación Media. Revista Electrónica Diálogos Educativos. 22 (11). pp. 33-65. 2011.

MARRERO, A. Introducción a la investigación en ciencias sociales. 1 ed. Reimpresión. Fundación de Cultura Universitaria. Montevideo-Uruguay. 2012.

MASSA, M. La enseñanza de las Ciencias Naturales en la Escuela Media: fundamentos y desafíos. En Massa, M., Foresi, M., F., y Sanjurjo, L. La Enseñanza de las Ciencias Naturales en la Escuela Media. (pp. 77-203). 1 ed. Rosario: Homo Sapiens Ediciones. 2015.

MINISTERIO DE EDUCACIÓN PÚBLICA. Programa de Estudio de Ciencias de Tercer Ciclo de la Educación General Básica. 2005.

MINISTERIO DE EDUCACIÓN PÚBLICA. Programa de Estudio de Ciencias de Tercer Ciclo de la Educación General Básica. 2017.

MOLINA, J., I. La educación en Costa Rica de la época colonial al presente. 1a ed. PEN. EDUPUC. San José, CR. 2016.

MORA V., A. Guía para elaborar una propuesta de investigación. Revista Educación, 29 (2), 67-97. 2005.

OSSENBACH, G.; SOMOZA, M. Los manuales escolares como fuente para la historia de la educación en América Latina. Editorial UNED. 2009.

OVARES, R. F. Educación como integración ideológica: lectura crítica de los textos ODECAROCAP. San José. Costa Rica. 1977. Recuperado de http://www.repositorio.una.ac.cr/bitstream/handle/11056/2105/recurso_202.pdf?sequence=1

PENZO, W., FERNÁNDEZ, V., GARCÍA, I., GROS, B., PAGÈS, T., ROCA, M., et al. Guía para la elaboración de las actividades de aprendizaje. Barcelona: Ediciones Octaedro. 2010.

PINTO, M. Calidad y evaluación de los contenidos electrónicos. 2015. Recuperado de http://www.mariapinto.es/e-coms/eva_con_elec.htm.

QUINTANILLA; M., CUÉlLAR; L.; CAMACHO, J. La historia del átomo en los libros de texto. Didáctica de una propuesta de innovación construida desde una visión naturalizada de la ciencia. III Jornada D'Història de la Ciència I Ensenyament. Nova Època. 1 (2). pp. 97-107. 2008.

RAMÍREZ, S., FLEISNER, A.; VIERA, L. Temas de química cuántica: análisis de su presentación en libros de texto de química general. Educación Química, 28(3), 147-153. 2017.

SÁEZ, R., I. Análisis de las actividades en libros de texto de Historia, Geografía y Ciencias Sociales de la educación básica en Chile. (Tesis Doctoral) Universidad de Barcelona, España. 2016.

SOUZA M., M., C. La artesanía de la investigación cualitativa. 1 ed. Buenos Aires. Lugar Editorial. 2009. 
VILLALOBOS, J. El docente y actividades de enseñanza/aprendizaje: algunas consideraciones teóricas y sugerencias prácticas. Educere, Artículos Arbitrados, 22, 170-176. 2003.

\section{Sobre os autores}

Adriana Gómez Jiménez. Licenciada en la Enseñanza de las Ciencias Naturales y Doctoranda en Educación en Ciencias Experimentales por la Universidad Nacional del Litoral, Argentina. Docente de Ciencias Naturales en la Educación Secundaria de Costa Rica. Correo Electrónico: adrianagj2001@gmail.com

Silvia Porro. Doctora en Ciencias Bioquímicas por la Universidad Nacional de La Plata, Argentina, y Especialista en Docencia en Entornos Virtuales por la Universidad Nacional de Quilmes (UNQ), Argentina. Profesora titular del Departamento de Ciencia y Tecnología de la UNQ. Directora del Grupo de Investigación en Enseñanza de las Ciencias (GIECIEN) y del proyecto Educación de las competencias científica, tecnológica y pensamiento crítico mediante la enseñanza de temas de naturaleza de ciencia y tecnología (CYTPENCRI). Correo Electrónico: porro@unq.edu.ar

Manuel Sandoval Barrantes. Doctor en Química Sostenible por la Universidad Complutense de Madrid, España. Presidente del Comité Organizador de la Olimpiada Costarricense de Química, Coordinador de la carrera de Ingeniería en Bioprocesos Industriales e investigador del Laboratorio de Investigación en Biorrefinería de la Escuela de Química de la Universidad Nacional en Costa Rica. Correo Electrónico: manuel.sandoval.barrantes@una.cr 